\title{
PROJECTION CONSTANTS FOR $C(S)$ SPACES WITH THE SEPARABLE PROJECTION PROPERTY
}

\author{
JOHN WARREN BAKER
}

\begin{abstract}
It is shown that if $n$ and $k$ are positive integers and $C\left(\omega^{n} k\right)$ is the Banach space of continuous functions on the compact set $I^{\prime}\left(\omega^{n} k\right)=\left\{\alpha \mid \alpha\right.$ is an ordinal, $\left.\alpha \leqq \omega^{n} k\right\}$ then $C\left(\omega^{n} k\right) \in P^{\prime}$ if and only if $\gamma \leqq 2 n+1$. This establishes the value of the projection constant for all $C(S)$ spaces possessing the separable projection property.
\end{abstract}

1. Introduction. A separable Banach space $X$ has the separable projection property if for every separable Banach space $Y$ and every isometric embedding $u: X \rightarrow Y$, there is a projection $\Pi$ of $Y$ onto $u(X)$. If $\Pi$ can always be selected with $\|\Pi\| \leqq \lambda, X$ is a $P_{\lambda}^{\prime}$ space (denoted $X \in P_{\lambda}^{\prime}$ ). The space $X$ has the separable extension property if for each separable Banach space $Y$ with $X \subset Y$ and each isomorphism $u$ of $X$ into some Banach space $B$, there is an extension $\bar{u}$, of $u$ from $Y$ into $B$. In [6], D. Dean showed that if $X$ has the separable projection property, it is a $P_{\lambda}^{\prime}$ space for some finite $\lambda$, and this property is equivalent to the separable projection property. D. Amir (see [1], [2]) has shown that if $S$ is a compact metric space, then $C(S)$ has the separable projection property if and only if $S$ is homeomorphic to the set $\left.\Gamma(\omega)^{n} k\right)$ of ordinals for some positive integers $n$ and $k$.

If a Banach space $X$ has the separable projection property, the number

$$
p_{s}(X)=\inf \left\{\lambda \geqq 1 \mid X \in P_{\lambda}^{\prime}\right\}
$$

will be called the (separable) projection constant. In [12], A. Sobczyk established $p_{s}\left(c_{0}\right)=2$ and R. McWilliams in [10] showed that $p_{s}(c)=3$. Recently, A. Pełczyński [11, p. 74] indicated it would be interesting to know the values of $p_{s}\left(C\left(\omega^{n}\right)\right)$ for $1 \leqq n<\omega$. Here we show $p_{s}\left(C\left(\omega^{n} k\right)\right)=$ $2 n+1$ for $1 \leqq n, k<\omega$. This establishes the values of the projection constant for all continuous function spaces with the separable projection property and includes McWilliam's result.

Received by the editors September 20, 1972 and, in revised form, October 13, 1972 and February 26, 1973.

AMS (MOS) subject classifications (1970). Primary 46B99, 46E15.

Key words and phrases. Banach spaces, continuous function spaces, separable projection property. 
2. Preliminaries. If $X$ is a topological space, a decomposition $D$ of $X$ is a disjoint collection of closed subsets of $X$ such that $X=\bigcup\{A: A \in D\}$. The notation $X / D$ denotes the set $D$ with its quotient topology. A set $A \in D$ is called plural if it contains at least two elements. Also, a set $A \in D$ is called a limit set if each open set containing $B$ has nontrivial intersection with a plural set in $D \sim\{B\}$. The $n$th derived decomposition $D^{(n)}$ of $X$ is defined as follows: $D^{(1)}$ is the decomposition of $X$ consisting of the plural limit sets in $D$ and singleton sets. Inductively, if $D^{(n)}$ is defined, then $D^{(n+1)}$ is the decomposition of $X$ consisting of the plural limit sets in $D^{(n)}$ and singleton sets. If $D^{(n)}$ contains no plural sets, we write $D^{(n)}=0$. The concept of the $n$th derived set is due to R. Arens [3]. A subset $Y$ of $X$ is $D$-saturated if it is a union of sets in D. Any additional terminology and the basic properties of decompositions used here may be found, for example, in [8].

If $X$ and $Y$ are compact Hausdorff spaces and $\phi$ is a (continuous) map of $X$ onto $Y$, then $\phi^{\circ}$ denotes the isometric isomorphism from $C(Y)$ into $C(X)$ that takes $f$ to $f \circ \phi$. If $Y=X / D$ and $\phi$ is the quotient map of $D$, then $\phi^{\circ}[C(X / D)]$ is identified with $C(X / D)$ and consists of all functions in $C(X)$ which are constant on each set in $D$. If $\lambda$ is an ordinal, the topological derivative of order $\lambda$ of $X$, denoted $X^{(\lambda)}$, is defined as follows: $X^{(0)}=X, X^{(\lambda+1)}=\left(X^{(\lambda)}\right)^{\prime}$, and $X^{(\lambda)}=\bigcap_{\beta . \lambda} X^{(\beta)}$ for $\lambda$ a limit ordinal, where $X^{\prime}$ denotes the derived set of $X$.

\section{Results.}

Lemma. If $H$ is a subspace of $C([0,1])$ isometrically isomorphic to $C\left(()^{n} k\right)$, then there is a projection $\mathrm{II}: C([0,1]) \rightarrow H$ with $\|\mathrm{II}\| \leqq 2 n+1$.

Proof. Let $u$ be an isometric isomorphism from $C\left(\omega^{n} k\right)$ onto $H$. By a theorem of W. Holsztyński (see [7] or [11]), there is a closed subset $Q$ of $[0,1]$, a map $\phi$ of $Q$ onto $\Gamma\left((1)^{n} k\right)$ and $\varepsilon$ in $C(Q)$ such that $|\varepsilon(q)|=1$ and $\varepsilon(q)(u g)(q)=\phi^{\circ} g(q)$ for all $q \in Q$ and $g \in C\left(\omega^{n} k\right)$. Let $D$ be the upper semicontinuous decomposition $\left\{\phi^{-1}(\alpha) \mid \alpha \in \Gamma\left(\omega^{n} k\right)\right\}$ of $Q$ induced by the closed map $\phi$. Define $Q_{1}=\phi^{-1}\left[0,\left(\omega^{n}\right]\right.$ and $\left.Q_{i}=\phi^{-1}\left(\omega^{n}(i-1) \text {, (1) }\right)^{n}\right]$ for $1 \leqq i<k$ and let $D_{i}$ be the restriction of $D$ to $Q_{i}$. Then $D_{i}$ is an u.s.c. decomposition of the compact set $Q_{i}$. If $H_{i}$ is the u.s.c. decomposition $D_{i}^{(1)}$ of $Q_{i}$, then $\left(\omega^{n}(i-1),\left(i^{n} i\right]^{(n+1)}=\varnothing\right.$ implies $H_{i}^{(n)}=0$. By Theorem 1.9 in [4], there is a projection $P_{i}$ of $C\left(Q_{i}\right)$ onto $C\left(Q_{i} / H_{i}\right)$ with $\left\|P_{i}\right\| \leqq 2 n+1$. Let $Y_{i}$ be the union of the plural sets in $D_{i}-H_{i}$. Each plural set $S$ in $D_{i}-H_{i}$ is open and closed in $Q_{i}$. Let $x(S)$ be a point in $S$ and for $f \in C\left(Q_{i}\right)$ define

$$
\begin{aligned}
P_{i}^{*} f(x) & =P_{i} f(x), & & \text { if } x \in Q_{i}-Y_{i} \\
& =P_{i} f(x(S)), & & \text { if } x \in S \subset Y_{i} .
\end{aligned}
$$


We show $P_{i}^{*} f \in C\left(Q_{i} / D_{i}\right)$. Since $P_{i}^{*} f$ is defined on $Q_{i}$ and constant on each set in $D_{i}$, it suffices to show $P_{i}^{*} f$ is continuous.

Suppose $x_{n} \in Q_{i}$ and $x_{n} \rightarrow x$. If $x_{n} \in Q_{i}-Y_{i}$ for each $n$, then $x \in Q_{i}-Y_{i}$ and $P_{i}^{*} f\left(x_{n}\right) \rightarrow P_{i}^{*} f(x)$ since $P_{i}^{*} f$ agrees with $P_{i} f$ on $Q_{i}-Y_{i}$. Thus, it suffices to suppose each $x_{n} \in Y_{i}$. If $x \in Y_{i}$, then $x \in S$ for some open set $S$ in $D$ and there exists $N>0$ such that $x_{n} \in S$ for $n \geqq N$. Then $P_{i}^{*} f\left(x_{n}\right)=$ $P_{i}^{*} f(x)$ for $n \geqq N$; so $P_{i}^{*} f\left(x_{n}\right) \rightarrow P_{i}^{*} f(x)$. Therefore, we may assume $x \in Q_{i}-Y_{i}$. Let $\delta>0$ and choose $S \in H_{i}$ with $x \in S$. Then $P_{i} f$ is constant on $S$. Thus there exists a $D_{i}$-saturated neighborhood $U$ of $S$ such that $\left|P_{i} f(y)-P_{i} f(x)\right|<\delta$ for all $y \in U$. There exists $N>0$ such that $x_{n} \in U$ for all $n \geqq N$. Therefore, if $x_{n} \in S_{n} \in D_{i}$, then $S_{n} \subset U$ and

$$
\left|P_{i}^{*} f\left(x_{n}\right)-P_{i}^{*} f(x)\right|=\left|P_{i} f\left(x\left(S_{n}\right)\right)-P_{i} f(x)\right|<\delta
$$

for all $n \geqq N$. Consequently, $P_{i}^{*} f$ is continuous. It follows that $P_{i}^{*}$ is a projection of $C\left(Q_{i}\right)$ onto $C\left(Q_{i} / D_{i}\right)$ with $\left\|P_{i}^{*}\right\| \leqq\left\|P_{i}\right\| \leqq 2 n+1$.

Since each $Q_{i}$ is both open and closed, $C(Q)=C\left(Q_{1}\right) \oplus C\left(Q_{2}\right) \oplus \cdots$ $\oplus C\left(Q_{k}\right)$. For each $f=f_{1}+f_{2}+\cdots+f_{k} \in C(Q)$ with $f_{i} \in C\left(Q_{i}\right)$, let $P f=P_{1} f_{1}+P_{2} f_{2}+\cdots+P_{k} f_{k}$. Then $P$ is a projection of $C(Q)$ onto $C(Q / D)$ with $\|P\| \leqq 2 n+1$. Let $R$ denote the restriction operator from $C(X)$ onto $C(Q)$ and $\varepsilon^{\prime}=1 / \varepsilon$. Define $\Pi=u\left(\phi^{\circ}\right)^{-1} P T_{\varepsilon} R$ where $T_{\varepsilon}: C(Q) \rightarrow C(Q)$ by $T_{\varepsilon} f=\varepsilon f$. Clearly $\Pi$ is a continuous linear operator from $C(X)$ into $H$. If $f \in H$, say $f=u g$, then $R F=f \mid Q=\varepsilon^{\prime} \phi^{\circ} g$. Therefore,

$$
\Pi f=u\left(\phi^{\circ}\right)^{-1} P T_{\varepsilon}\left(\varepsilon^{\prime} \phi^{\circ} g\right)=u\left(\phi^{\circ}\right)^{-1} P \phi^{\circ} g=u g=f .
$$

This shows $\Pi$ is a projection. Clearly, $\|\Pi\| \leqq 2 n+1$.

THEOREM. $\quad p_{s}\left(C\left(\omega^{n} k\right)\right)=2 n+1$.

Proof. Let $E$ be a separable Banach space, $S=\mathrm{\Gamma}\left(\omega^{n} k\right)$, and $u: C(S) \rightarrow E$ be an isometric embedding. By the Banach-Mazur theorem, we may assume $E$ is a subspace of $C([0,1])$. By the preceding lemma, there is a projection II of $C([0,1])$ onto $u[C(S)]$. The restriction $P$ of II to $E$ is a projection of $E$ onto $u[C(S)]$ with $\|P\| \leqq 2 n+1$; hence, $C(S) \in P_{2 n+1}^{\prime}$.

The fact that $C(S) \notin P_{\lambda}^{\prime}$ for $\lambda<2 n+1$ is established by Amir in the proof of the theorem in [1]. For completeness sake, we sketch his proof. Let $\Sigma$ be a countable field of subsets of $S$ which contains a basis for the open sets in $S$ and is closed under complements, finite unions, and the closure operation. Denote by $B(S, \Sigma)$ the closed subspace spanned in $m(S)$ by the characteristic functions of the sets of $\Sigma$. Then $B(S, \Sigma)$ is a separable Banach space containing $C(S)$. Since for each $n$-tuple $\left(k_{1}, k_{2}, \cdots, k_{n}\right)$ of positive integers, $S$ is $\left(k_{1}, k_{2}, \cdots, k_{n}\right)$ - $\sum$-connected (see [1] for definition) at $\omega^{n}$, it follows from the lemma in [1] that if $P$ is a projection of $B(S, \Sigma)$ onto $C(S)$, then $\|P\| \geqq 2 n+1$. Therefore, $C(S) \notin P_{\lambda}^{\prime}$ for $\lambda<2 n+1$. 


\section{BIBLIOGRAPHY}

1. D. Amir, Continuous function spaces with the separable projection property, Bull. Res. Counc. Israel, Sect. F 10F (1962), 163-164. MR 27 \#566.

2. - Projections onto continuous function spaces, Proc. Amer. Math. Soc. 15 (1964), 396-402. MR 29 \#2634.

3. R. Arens, Projections on continuous function spaces, Duke Math. J. 32 (1965), 469-478. MR 31 \#6108.

4. J. W. Baker, Some uncomplemented subspaces of $C(X)$ of the type $C(Y)$, Studia Math. 36 (1970), 85-103. MR 43 \#1113.

5. - Uncomplemented $C(X)$-subalgebras of $C(X)$, Trans. Amer. Math. Soc. (to appear).

6. D. Dean, Projections in certain continuous function spaces $C(H)$ and subspaces of $C(H)$ isomorphic with $C(H)$, Canad. J. Math. 14 (1962), 385-401. MR 26 \#1738.

7. W. Holsztyński, Continuous mappings induced by isometries of spaces of continuous functions, Studia Math. 26 (1966), 133-136. MR 33 \#1711.

8. J. Kelley, General topology, Van Nostrand, Princeton, N.J., 1955. MR 16, 1136.

9. R. McWilliams, On projections of separable subspaces of $(m)$ onto $(c)$, Proc. Amer. Math. Soc. 10 (1959), 872-876. MR 22 \#182.

10. A. Pełczyński, Linear extensions, linear averagings, and their applications to linear topological classification of spaces of continuous functions, Dissertationes Math. Rozprawy Mat. 58 (1968), 1-92. MR 37 \#3335.

11. 513-522. MR 38 \#2578.

12. A. Sobczyk, Projections of the space $(m)$ on its subspace $\left(c_{0}\right)$, Bull. Amer. Math. Soc. 47 (1941), 938-947. MR 3, 205.

Department of Mathematics, Florida State University, Tallahassee, Florida 32306 